\title{
Formation of silicon surface gratings with high-pulse-energy ultraviolet laser
}

\author{
Cheng-Yen Chen \\ Department of Electrical Engineering and Graduate Institute of Electro-Optical Engineering, \\ National Taiwan University, Taipei, Taiwan, Republic of China \\ Kung-Jeng Ma and Yen-Sheng Lin \\ Department of Mechanical Engineering, Chung Cheng Institute of Technology, Taoyuan, Taiwan, \\ Republic of China \\ Chee-Wee Liu, Chih-Wei Hsu, Chung-Yen Chao, Steffen Gurtler, and C. C. Yang ${ }^{\text {a) }}$ \\ Department of Electrical Engineering and Graduate Institute of Electro-Optical Engineering, \\ National Taiwan University, 1, Roosevelt Road, Sec. 4, Taipei, Taiwan, Republic of China
}

Received 2 December 1999; accepted for publication 12 July 2000)

We report the morphology, composition, and interaction mechanisms of silicon surface gratings fabricated with the fourth harmonic $(266 \mathrm{~nm})$ of a $Q$-switched Nd:YAG laser. We paid particular attention to the laser fluence dependence of silicon grating formation. It was found that at low fluence levels, grating formation was mainly caused by silicon oxidation. However, at high fluence levels gratings were formed with thermal ablation. In the former case, it was found that water vapor, instead of oxygen molecules, in the air was the key species providing oxygen for silicon oxidation. In the latter case, grating morphology was controlled by laser fluence level. These conclusions were supported by the measurement results of atomic force microscopy, energy-dispersive x-ray spectroscopy, Fourier-transform infrared spectroscopy, and chemical etching. The results of real-time monitoring of grating growth are also reported. (C) 2000 American Institute of Physics. [S0021-8979(00)07820-8]

\section{INTRODUCTION}

Surface and subsurface gratings have applications in various areas, including optical communications, display, storage, and sensing. The diffraction effects of a surface or subsurface grating can be used for the operations of various devices. Although gratings can be fabricated with etching techniques, such a process requires the preparation of a mask and photolithography techniques. They are usually quite complicated. Recently, because of the development of highpulse-energy and high-peak-power lasers, particularly in the UV range and in the femtosecond range, and related techniques, direct writing of surface or subsurface gratings with lasers has become an important alternative. ${ }^{1-10}$ Materials on which gratings were directly written included glass (including optical fiber) (e.g., Refs. 1-3), quartz, ${ }^{4} \mathrm{LiNbO}_{3},{ }^{5,6}$ silicon, ${ }^{7} \mathrm{GaAs} / \mathrm{AlGaAs},{ }^{8} \mathrm{GaN},{ }^{9}$ polymers (e.g., Ref. 10), etc. The mechanisms for forming either surface or subsurface gratings include the induced refractive index change by modifying material structures, such as bond breaking for fabricating fiber gratings (e.g., Refs. 1 and 2) and quantum well intermixing for fabricating semiconductor waveguide gratings. ${ }^{8}$ These mechanisms also include the physical and/or chemical interactions of the material surface with lasers. For such mechanisms, the used lasers are either in the UV range for the high-photon energy, including 193 and $248 \mathrm{~nm}$ excimer lasers, the third and fourth harmonics of a $Q$-switched Nd:YAG laser, or with femtosecond pulse widths for high

\footnotetext{
${ }^{a)}$ Electronic mail: ccy@cc.ee.ntu.edu.tw
}

peak power, such as an amplified mode-locked Ti:sapphire laser. Surface gratings formed with physical and/or chemical surface interactions are the major concern of this article.

Basically, writing surface gratings with laser is a process of exposing a sample to laser interference fringes. The photons at the bright lines of the fringes interact with the sample material to form periodical corrugations. Because we can control the grating period through interference arrangement and the corrugation depth through laser power level, fabrication of surface grating with laser is more flexible than other techniques. Since all the materials mentioned above, except quartz, have quite large absorption coefficients at the UV range, melting and related processes are the key mechanisms responsible for the grating formation. In this article, we report the morphology, composition, and interaction mechanisms of silicon surface gratings fabricated with the fourth harmonic $(266 \mathrm{~nm})$ of a $Q$-switched Nd:YAG laser. In particular, we studied the laser fluence dependence of silicon grating formation. We found that at low fluence levels, grating formation was mainly caused by silicon oxidation. However, at high fluence levels gratings could be formed with thermal ablation. In the former case, it was found that water vapor, instead of oxygen molecules, in the air was the key species providing oxygen for silicon oxidation. In the latter case, grating morphology was controlled by laser fluence. The results of real-time monitoring of grating growth will also be reported.

In Sec. II of this article the optical systems used for grating formation and characterization are described. The results of silicon gratings formed with the oxidation process at 


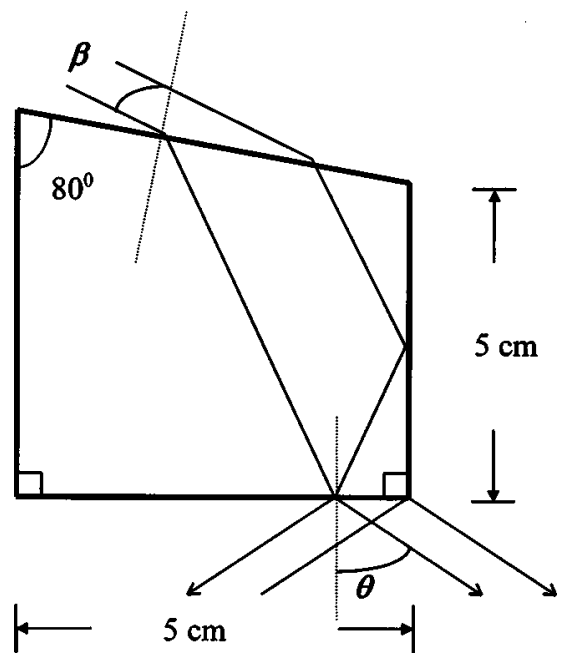

FIG. 1. Geometry of the prism for forming the interference fringe at the lower-right corner. The fringe period and size are controlled by the incident angle $\beta$.

low laser fluence levels are discussed in Sec. III. Then, realtime monitoring of grating formation is reported in Sec. IV. Here, gratings formed with thermal ablation, instead of silicon oxidation, at high laser fluence levels are introduced. Morphology study of the gratings formed with single-shot, high-fluence laser pulse is discussed in Sec. V. Finally, conclusions are drawn in Sec. VI.

\section{OPTICAL SYSTEMS FOR GRATING FORMATION AND CHARACTERIZATION}

We used two optical systems for grating formation and real-time monitoring the growth process. In the first system, a $5 \mathrm{~cm} \times 5 \mathrm{~cm}$ prism with the geometry shown in Fig. 1 was used. When a laser beam was incident from the upper-left corner, as shown with the two parallel lines, it was designed to let the beam center hit the lower-right corner. In this situation, one half of the laser beam was totally reflected from the prism side represented by the vertical line. This one-half beam overlapped with the other half to form an interference fringe. The fringe period was related to the angle $\theta$ and controlled by the incident angle $\beta$ and the prism dimensions. It could be adjusted from 180 to $600 \mathrm{~nm}$. By placing a silicon sample, contacting the bottom face of the prism near the corner, surface gratings could be formed. We used the fourth harmonic of a computer-controlled $Q$-switched Nd:YAG laser (Coherent, Infinity) for producing the interference fringes. The laser pulse width was $3.5 \mathrm{~ns}$. Because the laser is seeded with a diode-pumped source, its line width is quite narrow $\left(0.002 \mathrm{~cm}^{-1}\right)$ and its coherence length is as long as a few meters. Hence, it was quite simple to build the interferometers. Figure 2 shows the optical setup with the prism for writing gratings. After two cylindrical lenses, the laser beam was focused into an elliptical shape of $2 \mathrm{~cm} \times 0.5 \mathrm{~cm}$. Typically a grating width of $5 \mathrm{~mm}$ could be obtained. Depending on the grating period, the grating length was ranged from 12 to $37 \mathrm{~mm}$. A larger grating period corresponded to a smaller grating length. A fine rotator was used to carry the prism and control the grating period.

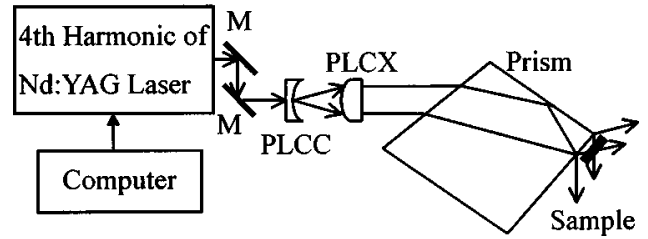

FIG. 2. The first optical setup for grating formation with the prism shown in Fig. 1.

For conducting real-time monitoring of grating growth, we prepared another optical system with its setup shown in Fig. 3. Here, we arranged a Mach-Zehnder interferometer (with the optical paths shown with the thick dotted lines) for forming the interference fringes inside a chamber. By rotating the mirrors $\mathrm{M} 3$ and M4, the period of the interference fringe could be varied from $300 \mathrm{~nm}$ to a few micrometers. Designated flown gas species through the chamber provided controlled ambient conditions. Interference fringes passed through the quartz window of the chamber with insignificant attenuation to write gratings on silicon samples $[p$ type, (100) orientation] mounted inside the chamber. A HeNe laser at $632.8 \mathrm{~nm}$ was used to monitor the reflected (the zerothorder diffracted) and the first-order diffracted intensities. Such optical paths are shown in Fig. 3 with thin dotted lines. The reflected and diffracted powers were detected by two photodetectors, which were connected to an oscilloscope and a computer.

\section{SILICON GRATING FORMED WITH OXIDATION PROCESS}

It was discovered that when a silicon grating was formed with relatively low-pulse energy, the formation of grating was due to gradual oxidation of silicon. When a silicon sample was exposed to an interference fringe, the locations of bright lines became oxidized. Because silicon oxide has a larger volume compared with pure silicon, these locations swelled to become crests of the grating pattern. In this pro-

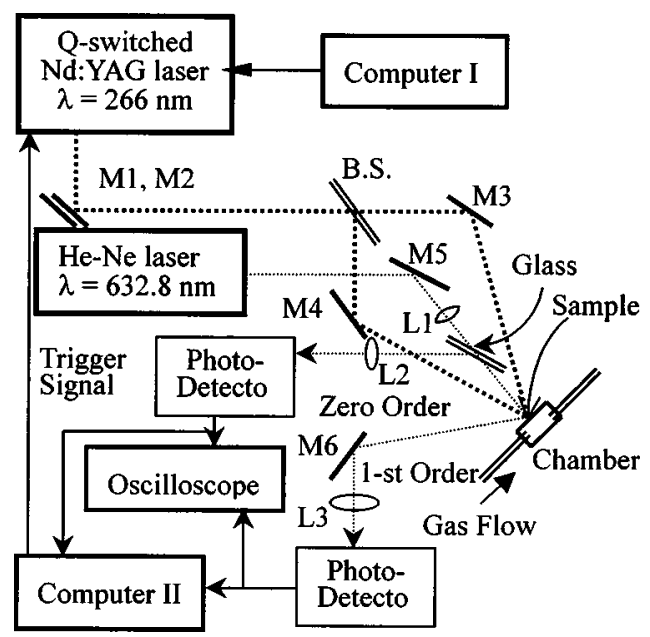

FIG. 3. The second optical setup for grating formation and real-time monitoring of grating growth with the samples in controlled ambient gases. The $\mathrm{HeNe}$ laser is used for real-time monitoring the reflected and diffracted signals from a formed grating. 

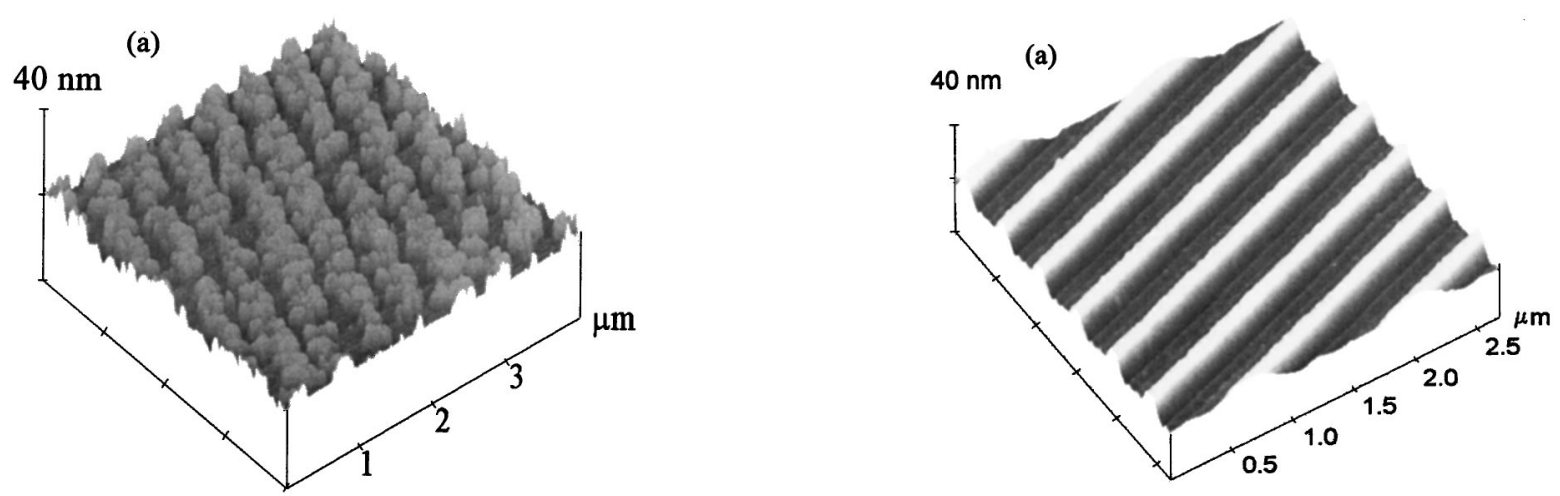

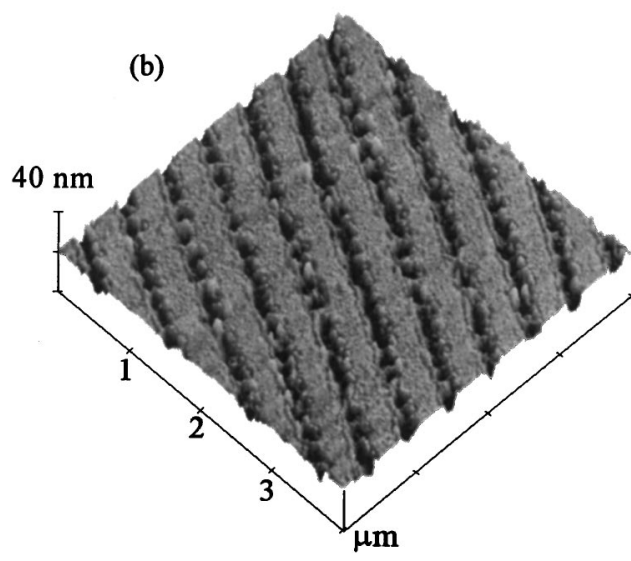

FIG. 4. AFM pictures of a grating formed with $84 \mathrm{~mJ} / \mathrm{cm}^{2}$ at $50 \mathrm{~Hz}$ for $50 \mathrm{~s}$. (a) and (b) refer to the grating morphology before and after HF etching, respectively.

cess, silicon was first melted by absorbing incident photons. Then, water or oxygen molecules diffused into the melted silicon to form silicon oxide. Since the melting temperature, latent heat, and heat capacity of crystalline silicon are 1685 $\mathrm{K}, 86 \mathrm{~kJ} / \mathrm{mole}$, and $34 \mathrm{~kJ} /($ mole $\mathrm{K})$, the minimum energy to melt silicon from the room temperature is about 133 $\mathrm{kJ} / \mathrm{mole}^{11}$ To estimate the minimum laser fluence for melting silicon, the heating depth must be determined first. From numerical simulation ${ }^{12}$ and theoretical prediction, ${ }^{13}$ the heating depth is the larger of the absorption depth and the thermal diffusion length $\left((2 D \tau)^{1 / 2}\right.$, where $D$ is the thermal diffusivity and $\tau$ is the pulse width), which are $5^{11}$ and $290 \mathrm{~nm}$, respectively, under the experimental conditions $(D=0.12$ $\mathrm{cm}^{2} / \mathrm{s}$ near the melting temperature, ${ }^{11}$ and $\tau=3.5 \mathrm{~ns}$ ). Hence, with the refractive index 1.916 at $266 \mathrm{~nm}$ at the room temperature, ${ }^{11}$ the minimum laser fluence for melting silicon is about $330 \mathrm{~mJ} / \mathrm{cm}^{2}$ under the assumption that silicon within the thermal diffusion length melts completely. This fluence level for a melting silicon surface is overestimated since a silicon surface melts before bulk silicon within the thermal diffusion length does. Figure 4(a) shows the atomic force microscopy (AFM) picture of a silicon grating formed with an average fluence level of $84 \mathrm{~mJ} / \mathrm{cm}^{2}$ and a pulse repetition frequency of $50 \mathrm{~Hz}$ for $50 \mathrm{~s}$. The grating was fabricated with the optical system shown in Fig. 2. From the AFM picture, we can see rows of cotton-shape structures with the row separation the same as the designated fringe period at 400 $\mathrm{nm}$. It is believed that these structures were created through

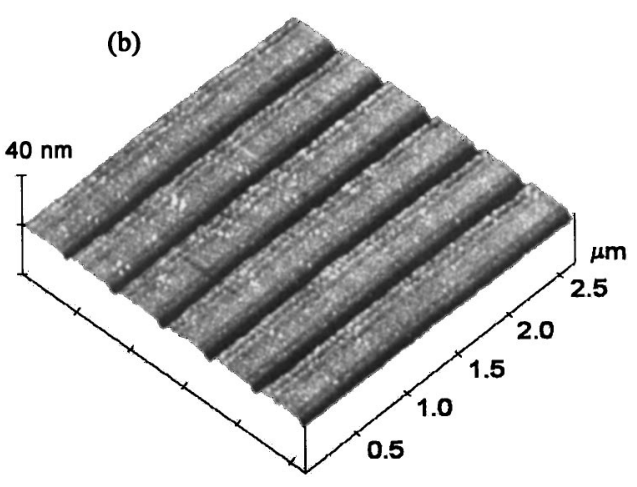

FIG. 5. AFM pictures of a grating formed with $168 \mathrm{~mJ} / \mathrm{cm}^{2}$ at $50 \mathrm{~Hz}$ for $50 \mathrm{~s}$. (a) and (b) refer to the grating morphology before and after HF etching, respectively.

silicon oxidation and corresponded to the bright lines of the interference fringe. To verify this hypothesis, we dipped the sample in an HF solution (10\% HF) for $30 \mathrm{~s}$. Since the HF etching rate of silicon oxide is much higher than that of silicon, we found that the cotton-shape structures were removed during etching, as shown in the AFM picture of Fig. 4(b). Here, we can see that the crests become valleys after HF etching. The comparison between Figs. 4(a) and 4(b) provides proof for silicon oxidation in the formation of grating crests. Also, it shows that silicon oxide grows into the sample beyond the level of the original valleys. Note that the average fluence level at $84 \mathrm{~mJ} / \mathrm{cm}^{2}$ mentioned above resulted from the average over the bright and dark lines of the interference fringe and over the nonuniform laser beam intensity. Therefore, the local fluence at the bright lines near the laser beam center should be higher than $330 \mathrm{~mJ} / \mathrm{cm}^{2}$, the overestimated critical fluence for a melting silicon surface.

To see the effects of relatively higher laser pulse energy, we increased the average fluence level to $168 \mathrm{~mJ} / \mathrm{cm}^{2}$ with the same pulse repetition rate and exposure time $(50 \mathrm{~Hz}$ for $50 \mathrm{~s})$. Figures 5(a) and 5(b) show the AFM results of this silicon grating. Again, the crests became valleys after HF etching. It is noted that the regularity of grating structure is much improved, compared with the case in Fig. 4. Here, a high-quality grating with the corrugation depth of around 15 $\mathrm{nm}$ and about $50 \%$ duty cycle was obtained [see Fig. 5(a)]. Also, note that a $<100 \mathrm{~nm}$ feature was formed after HF etching.

We also used energy dispersive x-ray (EDX) spectroscopy to verify the existence of oxygen atoms in the grating 


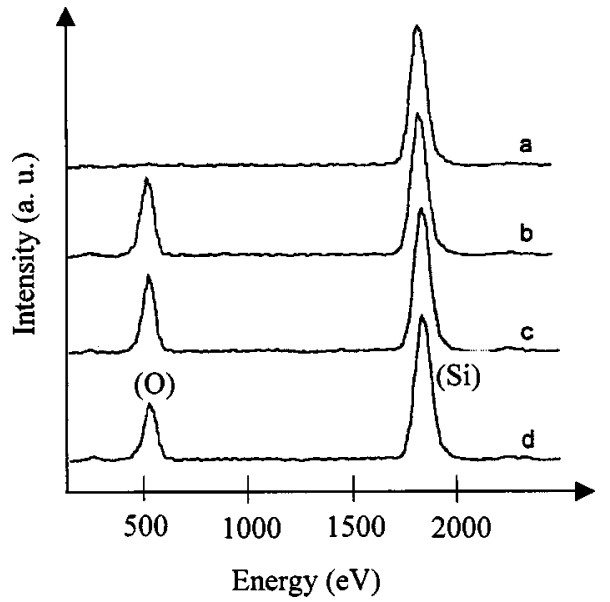

FIG. 6. EDX spectra of the grating of Fig. 5 before HF etching. Curves (a)-(d) represent the results of the untreated sample (a), the spatial average of the grating (b), the crest (c), and the valley (d) of the grating, respectively.

structures. Figure 6 shows the EDX spectra of the grating sample of Fig. 5 before HF etching. Spectrum (a) represents the result of an untreated silicon wafer. Spectrum (b) shows the result of a grating with an EDX electron spot much larger than the grating period. Therefore, it stands for the average result of crests and valleys. Here, one can see the appearance of an oxygen element (at $532 \mathrm{eV} \mathrm{x}$-ray energy) besides silicon (at $1839 \mathrm{eV}$ x-ray energy). Then, Spectra (c) and (d) were obtained with a high EDX resolution focused at a grating crest and valley, respectively. We can see that relatively more oxygen atoms exist in crests than valleys. This confirms the hypothesis of silicon oxidation at the bright lines of the fringe. The existence of little carbon content (the small feature left to the oxygen peak) is attributed to the incorporation of $\mathrm{CO}_{2}$ molecules in the air during laser illumination.

To understand the origin of oxygen for oxidation, we used the optical system in Fig. 3 for grating formation with controlled ambient gas. The three spectral curves in Fig. 7

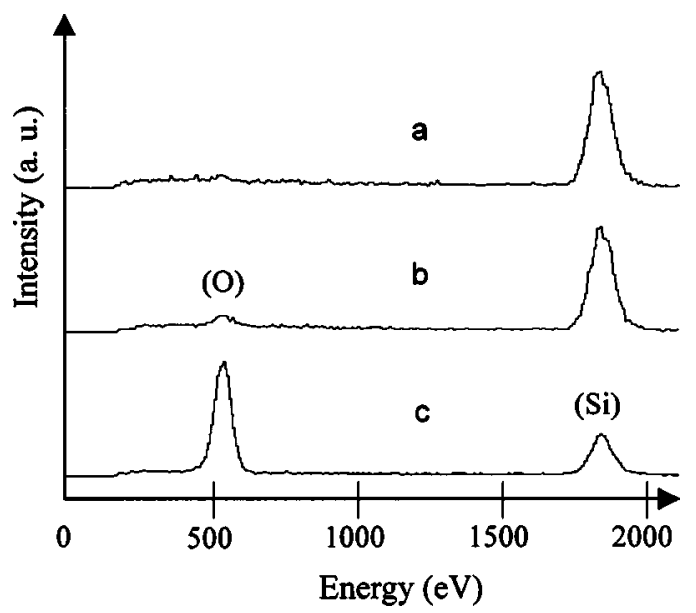

FIG. 7. EDX spectra of two samples processed in different ambient gases with the laser conditions as $168 \mathrm{~mJ} / \mathrm{cm}^{2}$ at $25 \mathrm{~Hz}$ for $120 \mathrm{~s}$. Curve (a) represents the result of an untreated silicon wafer. Curves (b) and (c) stand for those of the samples treated in ambient oxygen molecules and ambient air, respectively.

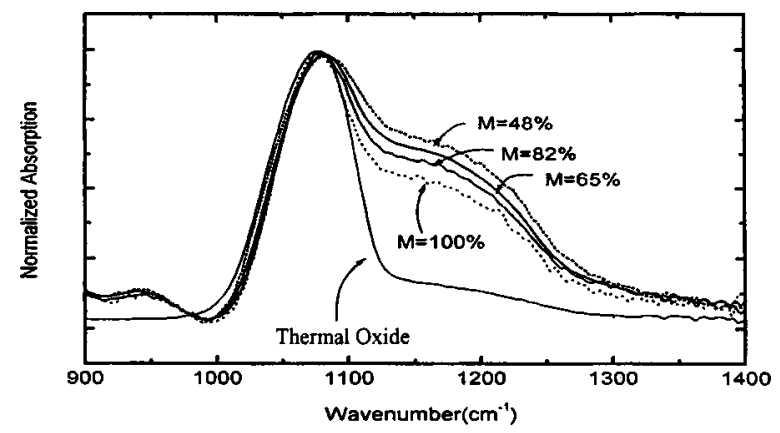

FIG. 8. FTIR absorption spectra of four gratings made under different conditions of humidity $M$. All samples were made at $20^{\circ} \mathrm{C}$ with the laser conditions of $480 \mathrm{~mJ} / \mathrm{cm}^{2}$ at $25 \mathrm{~Hz}$ for $200 \mathrm{~s}$. The result of thermal oxide is also shown for comparison.

show the EDX results of an untreated sample (a), a sample under fringe exposure in ambient oxygen molecules (around $1 \mathrm{~atm}$.) (b), and a sample under fringe exposure in ambient water vapor (air) (c). The laser exposure conditions for spectra (b) and (c) were the same: $168 \mathrm{~mJ} / \mathrm{cm}^{2}$ of $25 \mathrm{~Hz}$ for $120 \mathrm{~s}$. The comparison between spectra (b) and (c) shows that oxidation in ambient $\mathrm{O}_{2}$ was much weaker than that in ambient air or water vapor. Diffraction measurement also confirmed that grating was not formed in ambient $\mathrm{O}_{2}$. The relatively higher oxygen (than silicon) content in spectrum (c), when compared with spectra (b)-(d) in Fig. 6, was attributed to the thicker oxidation layer under these fabrication conditions. The much more active interaction of $\mathrm{H}_{2} \mathrm{O}$ with melted silicon to form silicon oxide, compared with $\mathrm{O}_{2}$, can be attributed to the polarization of $\mathrm{H}_{2} \mathrm{O}$ molecules. The polarization of a molecule makes it easier to be adsorbed by the melted silicon. ${ }^{14}$ Compared with $\mathrm{H}_{2} \mathrm{O}$, the unpolarized $\mathrm{O}_{2}$ molecules have much less adsorption to the melted silicon. The other possible reason for the stronger $\mathrm{H}_{2} \mathrm{O}$ interaction is that the existence of hydrogen atoms can create a pathway for enhancing the diffusion of oxygen atoms in melted silicon. ${ }^{15}$ Hence, in ambient $\mathrm{O}_{2}$ silicon grating could not be formed with the laser fluence range mentioned above. The difference between $\mathrm{H}_{2} \mathrm{O}$ and $\mathrm{O}_{2}$ in such an oxidation process deserves further investigation.

The oxidation process was incomplete. Figure 8 shows the Fourier transform infrared spectroscopy (FTIR) results of silicon gratings fabricated under various ambient conditions of humidity at $20^{\circ} \mathrm{C}$ (with the same laser conditions: 420 $\mathrm{mJ} / \mathrm{cm}^{2}$ at $25 \mathrm{~Hz}$ for $200 \mathrm{~s}$ ). We can see that on the largewave number side of the $\mathrm{SiO}_{2}$ characteristic absorption line at $1080 \mathrm{~cm}^{-1}$ (corresponding to the $\mathrm{Si}-\mathrm{O}$ stretching mode), there exists a broad shoulder. This shoulder is particularly clear when compared with the curve of thermal oxide, i.e., pure $\mathrm{SiO}_{2}$. Such an absorption shoulder was attributed to the existence of some kinds of $\mathrm{Si}-\mathrm{O}$ complexes. ${ }^{16,17}$ Our experiments showed that the absorption shoulder became weaker when the ambient humidity $M$ increased. In other words, more supply of water vapor leads to more complete oxidation. 

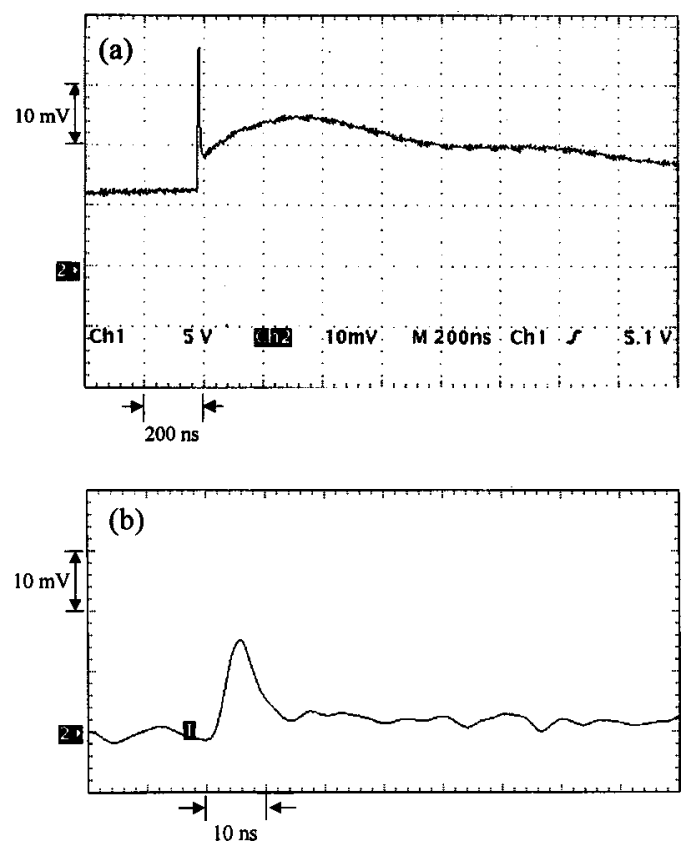

FIG. 9. Time evolution of the reflected (a) and diffracted (b) intensities during the process of single laser pulse exposure. The sharp peak in either part of the figure represents the generation of plasma.

\section{REAL-TIME MONITORING OF GRATING FORMATION}

To real-time monitor the growth process of grating during UV laser exposure, we used a $\mathrm{HeNe}$ laser to detect the reflected and diffracted signals as shown in Fig. 3. Figures 9 (a) and 9(b) demonstrate the temporal variations of reflection and diffraction intensities, respectively, after the exposure of one laser pulse of $204 \mathrm{~mJ} / \mathrm{cm}^{2}$ in average fluence and $3.5 \mathrm{~ns}$ in pulse width. In Fig. 9(a), the sharp peak of reflec- tion is supposed to be due to the stage of plasma generation after the heating and melting of silicon. ${ }^{18}$ In other words, in this stage plenty of electrons and holes were generated such that reflectivity was increased from the conducting surface. This stage lasts for around $10 \mathrm{~ns}$. The long tail of reflection after the plasma stage is attributed to silicon melting. After several $\mu$ s, oxidized silicon resolidifies. After resolidification with the illumination of a number of laser pulses, the reflectivity was reduced because of the resultant nonsmooth surface. Figure 9(b) shows the diffracted intensity. Here, we can see that after the hump, which corresponds to the plasma stage, the diffraction intensity level becomes higher, compared with the level before the hump. This is clear evidence of grating formation. The grating structure still existed after the melted silicon oxide resolidified.

We also monitored the diffracted intensity as a function of exposure pulse number during grating formation. The four parts of Fig. 10 show the evolution of diffracted signal intensities at four different laser fluence levels when gratings were fabricated in the air. At $204 \mathrm{~mJ} / \mathrm{cm}^{2}$, from part (a) we can observe silicon oxidation as the major mechanism for grating formation. One can see that significant diffraction is observed after 500 laser pulses exposure with the used figure scale. After that, the diffraction intensity grows quickly. However, after a maximum is reached, it starts to decrease. The decrease is attributed to the instability of the laser beam. A slight shift in beam position may change the positions of bright and dark lines of the interference fringe. To take a closer look at the growth rate of grating, in Fig. 11 we show the early stage of the evolution of diffraction intensity at two laser fluence levels: 174 and $204 \mathrm{~mJ} / \mathrm{cm}^{2}$. One can see that a higher fluence level leads to earlier appearance of a diffracted signal and a steeper increase of its intensity. Although there exist fluctuations, the growth basically follows
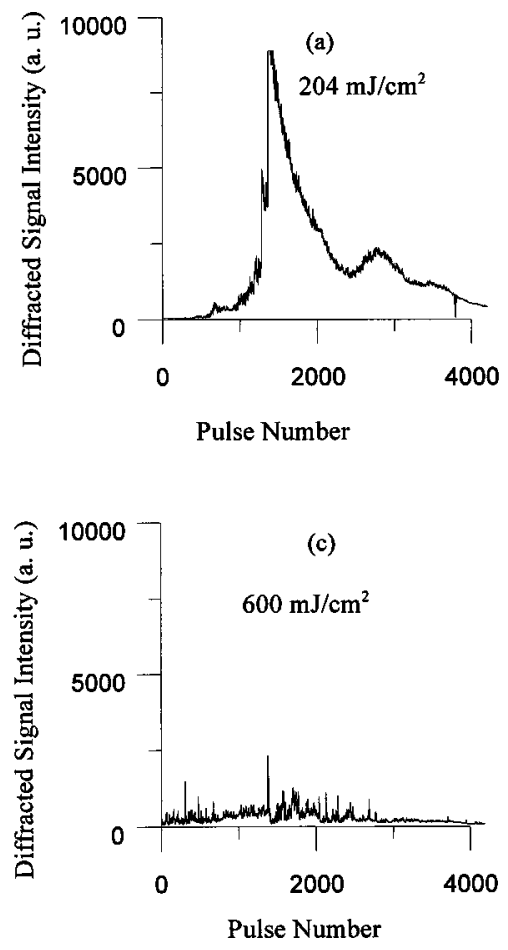
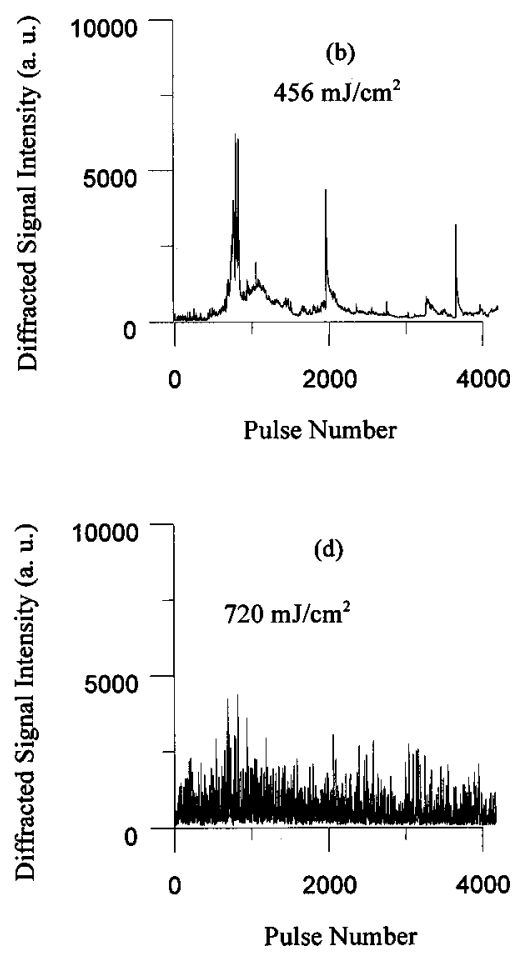

FIG. 10. Diffracted intensities as functions of the exposed pulse number in ambient air with different fluence levels. The grating formation mechanisms at low and high fluence levels are different. 


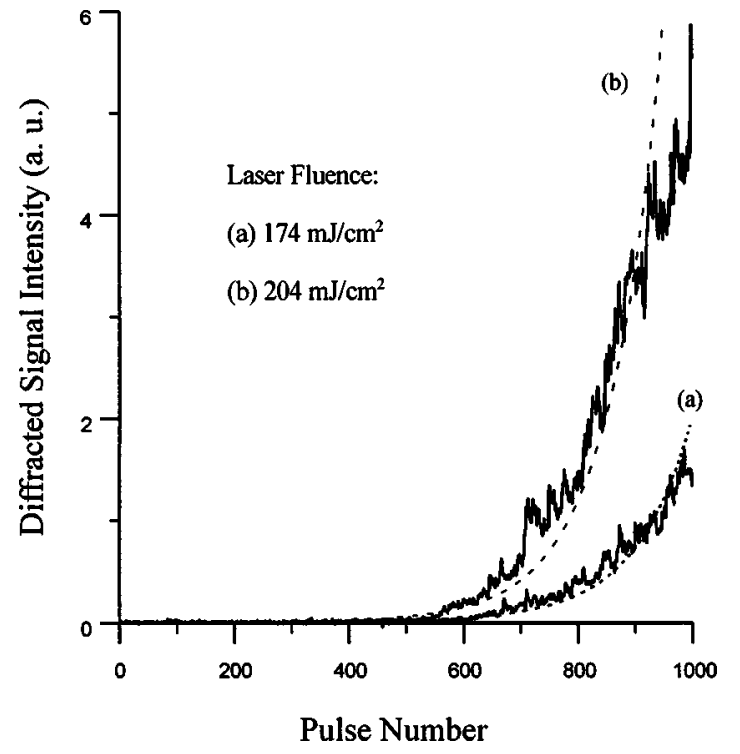

FIG. 11. A close look of the diffracted intensities as functions of the exposed pulse number in ambient air with two relatively low fluence levels. The grating formation is caused by the oxidation process.

exponential functions, as indicated by the fitting dashed curves in Fig. 11.

Now, we return to Fig. 10. Here, when the fluence increased to $456 \mathrm{~mJ} / \mathrm{cm}^{2}$ (with the maximum local fluence higher than $1600 \mathrm{~mJ} / \mathrm{cm}^{2}$ ), the construction and destruction of gratings became faster. Meanwhile, the maximum diffraction intensity was reduced. These phenomena became more prominent as the fluence level further increased. When the fluence was beyond $600 \mathrm{~mJ} / \mathrm{cm}^{2}$, random sharp spikes of diffraction intensity were observed. They implied the possi- bilities of formation and destruction of gratings within a few laser shots. It was speculated that the grating formation mechanism at this high fluence level was different from silicon oxidation. To confirm this speculation, we etched the formed grating with HF solution and found that the morphology was not changed after etching. Therefore, another mechanism is responsible for the formation of grating with high laser fluence levels. Further proof is provided with Fig. 12. Here, gratings were fabricated in ambient nitrogen. In this case, without any oxygen content silicon oxidation could not occur. One can see that no diffraction signal was observed at $204 \mathrm{~mJ} / \mathrm{cm}^{2}$. Beyond this level, the evolution of diffraction intensity was very similar to the case of ambient air. Such fast construction and destruction processes are supposed to be caused by thermal ablation. In other words, during the exposure to a laser pulse, silicon is first melted. Then, the fluid dynamics determines the morphology of grating structure (to be discussed in detail in Sec. V). In a medium fluence range, an induced shear force pushes the melted silicon away from the center of a fringe bright line to form a valley. The valley formation of this process may be cancelled by the oxidation process, which leads to crests at the centers of fringe bright lines, particularly at a medium fluence level. It is believed that the results of Fig. 10 with fluence levels between 456 and $600 \mathrm{~mJ} / \mathrm{cm}^{2}$ are involved in such a counteraction.

Note that the evaporation temperature and latent heat of silicon are $3060 \mathrm{~K}$ and $450 \mathrm{~kJ} / \mathrm{mole}$, respectively. ${ }^{11}$ Also, the heat capacity of liquid silicon is $25 \mathrm{~J} /($ mole $\mathrm{K})$. We have estimated the required $266 \mathrm{~nm}$ laser fluence for evaporating silicon to give around $1600 \mathrm{~mJ} / \mathrm{cm}^{2}$ by approximating the melting depth as the thermal diffusion length $(290 \mathrm{~nm})$. The

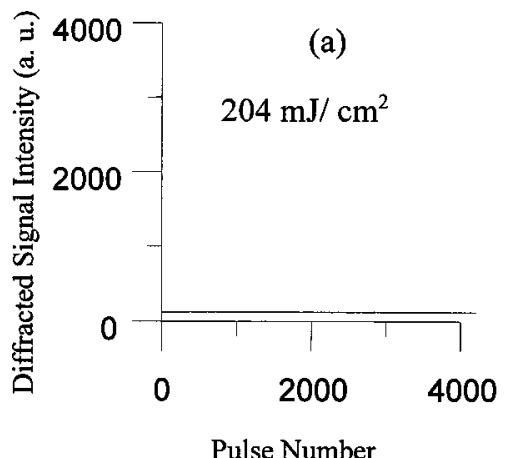

Pulse Number

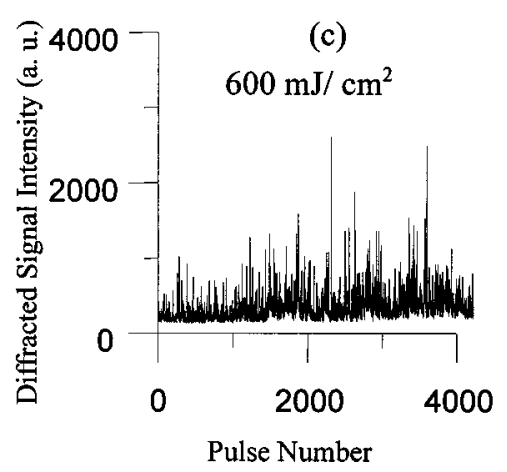

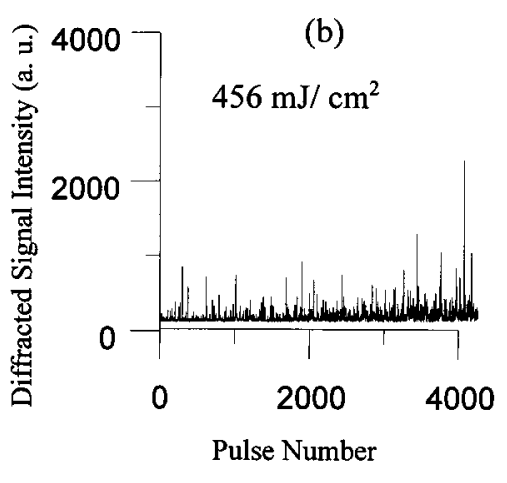

(d)

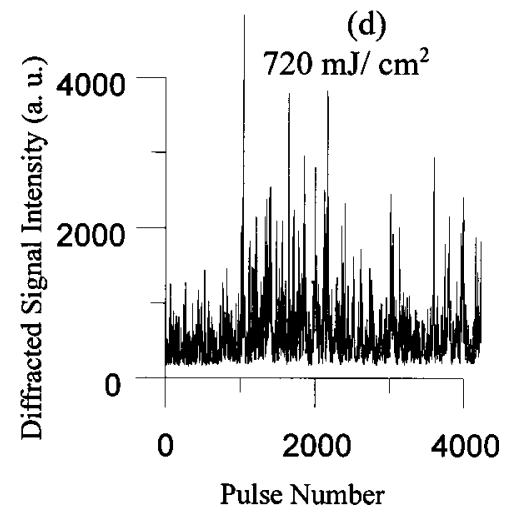

FIG. 12. Diffracted intensities as functions of the exposed pulse number in ambient nitrogen with different fluence levels. The oxidation process cannot occur in this situation. Gratings are formed with thermal ablation at high fluence levels. 

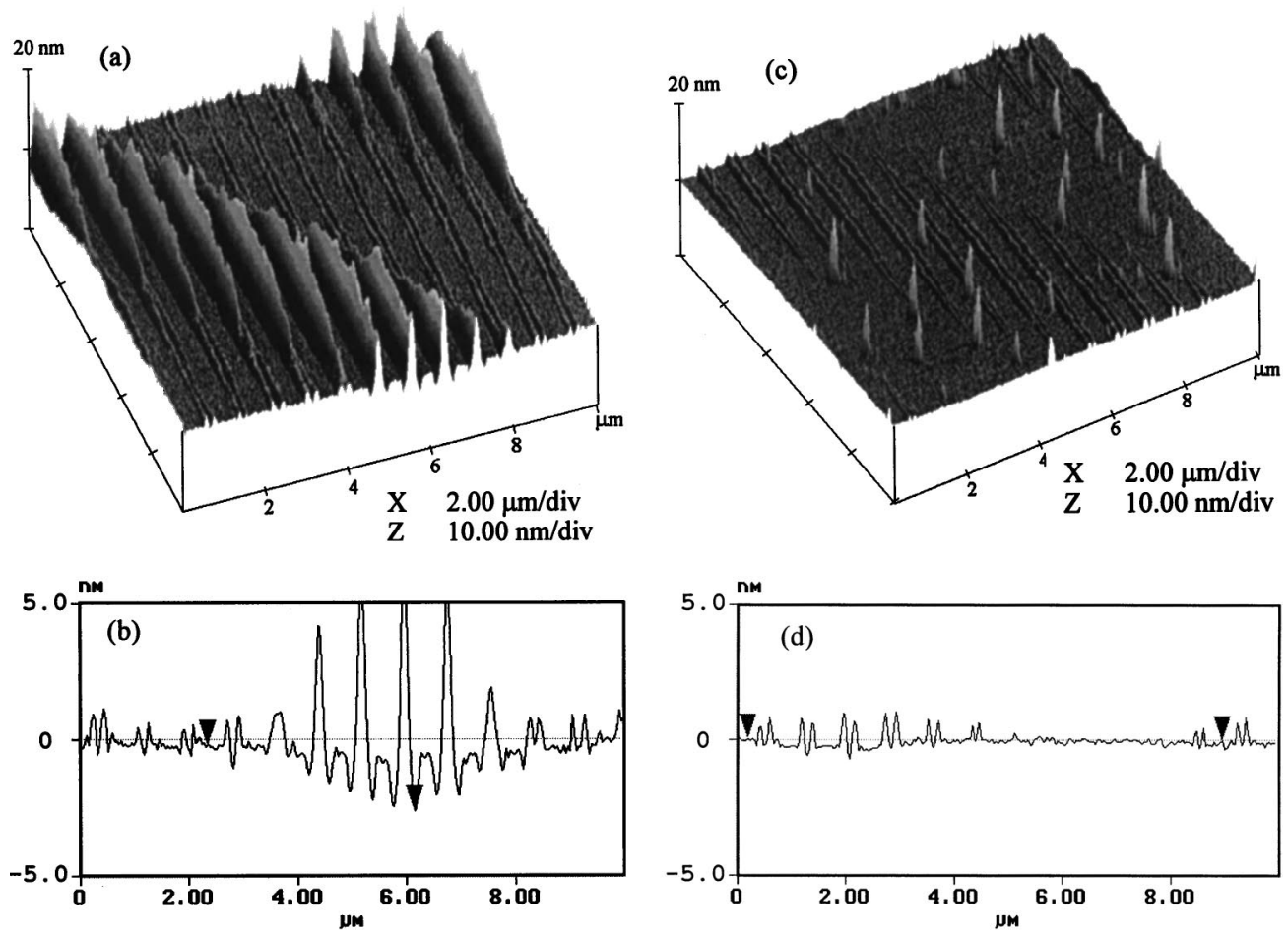

FIG. 13. Three-dimension and side-view AFM pictures of a grating formed with single laser pulse exposure at $840 \mathrm{~mJ} / \mathrm{cm}^{2}$. A large-period interference fringe of around $6 \mu \mathrm{m}$ in period is formed besides the designated grating period at about $800 \mathrm{~nm}$. (a) and (b) show the 3D picture and its side view in the portions of high and medium fluence levels. (c) and (d) show those in the portions of medium and low fluence levels.

local fluence levels of constructive interference (several times the given average values) for parts (b), (c), and (d) of Figs. 10 and 12 were well above this threshold value. Hence, evaporation might also occur in the aforementioned process. Nevertheless, we believe that the observed phenomena described above were essentially due to the silicon melting process, instead of evaporation.

\section{GRATING MORPHOLOGY FORMED WITH HIGH LASER FLUENCE}

For understanding the morphology of grating formed with a high fluence level, we used the single-shot operation of the UV laser to fabricate gratings. To create different fluence levels in a small area (smaller than the typical scanning range of AFM) within the laser beam, we slightly tilted the mirror M3 in the UV interferometer in Fig. 3 so that a phase gradient was generated over the laser beam. With this arrangement, besides the fringe of the designated period (around $800 \mathrm{~nm}$ ), there was another interference fringe of a larger period (around $6 \mu \mathrm{m}$ ) in the formed interference pattern. Figures 13(a) and 13(c) show the three-dimensional (3D) AFM pictures of a grating fabricated in this manner. They correspond to portions of the beam center and an edge, respectively. The spiky features in Fig. 13(c) were caused by dusts on the sample during AFM scanning. The grating was fabricated with a single-shot pulse exposure with an average fluence of $840 \mathrm{~mJ} / \mathrm{cm}^{2}$. In Fig. 13(a), the fringe of the larger period can be clearly seen. In the bright zones of this fringe, we can see the feature of a single hump with a small valley on either side at the bright lines of the small-period fringe.
However, in the dark zones of the large-period fringe, we see the feature of two humps with a valley at the center at the bright lines of the small-period fringe. These features can be more clearly seen in the sideview picture shown in Fig. 13(b). Then, at an edge of the laser beam (with a smaller fluence level) we see the double-hump feature in the bright zones and no feature in the dark zones of the large-period fringe. Again, Fig. 13(d) shows the side view.

The features described above can be summarized as follows: At a certain high fluence level, single-shot laser exposure can create a double-hump feature. However, at a higher fluence level, it produces a single-hump feature. Such laser fluence dependent morphology has been observed in thermal ablation of other materials. ${ }^{19-23}$ In the literature, researchers were dealing with thin films, such as $\mathrm{Te}-\mathrm{Se}-\mathrm{I}$ and $\mathrm{Ni}-\mathrm{P}$, and polymers. Typically, they observed a single-hump feature at a certain high fluence level. As the fluence increases, a hole was formed at the center of the hump, similar to the double-hump feature we observed. Such fluence dependent phenomena were interpreted as the counteraction of two forces. When the bright line of the interference fringe irradiates a silicon sample, a shear force is produced to push the melted silicon aside from the center. ${ }^{19}$ On the other hand, the surface tension tends to make the liquid silicon swell to form a hump. ${ }^{19,20}$ This is particularly true because the melted silicon may become amorphous after it resolidifies. The counteraction of the two trends determines the morphology of the resolidified structure. The result may also depend on the material properties of the sample, such as viscosity and specific heat. In our case with silicon, a higher fluence level leads to 
a thicker melted layer and hence possibly stronger surface tension and subsurface structure distortion. Hence, a singlehump feature is formed. On the other hand, a relatively lower fluence level results in a thinner melted layer and possibly a stronger out-push shear force. Therefore, a two-hump feature is produced. Such an interpretation might be oversimplified. Further investigation is required.

\section{CONCLUSIONS}

In summary, we have fabricated silicon surface gratings by exposing the samples to the interference fringes of the fourth harmonic of a $Q$-switched Nd:YAG laser. We found that the grating formation mechanisms with low and high laser fluence levels were quite different. With low fluence levels, the grating crests were produced with silicon oxidation. With high fluence levels, thermal ablation was the major mechanism for grating formation. The oxidation process was caused by the incorporation of $\mathrm{H}_{2} \mathrm{O}$ (instead of $\mathrm{O}_{2}$ ) into the melted silicon for incomplete oxidation. This process was verified with the HF etching, EDX, and FTIR measurements. At high fluence levels, with the thermal ablation process the morphology of a formed grating depends on the fluence level. The results of real-time monitoring of grating formation were also discussed.

From the practical viewpoint, silicon surface gratings should find applications in integrated circuit process technology. ${ }^{7}$ For this goal, laser beam stability and hence the growth rate need to be well controlled. We have checked the structure of resolidified silicon with high-resolution transmission electron microscopy. It showed that a single crystalline structure was retained below the oxidized layer. Such material structures must be good enough for optoelectronics applications. On the other hand, from the viewpoint of basic research the details of the oxidation process and thermal ablation require further investigation. In particular, the fundamental mechanisms of incomplete silicon oxidation and the formation of either single- or double-hump morphology are important issues. These topics represent our directions of further research efforts.

\section{ACKNOWLEDGMENT}

This research was supported by the National Science Council, The Republic of China, under Grants No. NSC 882112-M-002-004, No. NSC 88-2215-E-002-012, and No. NSC 88-2215-E-002-014.

${ }^{1}$ K. O. Hill, Y. Fujii, D. C. Johnson, and B. S. Kawasaki, Appl. Phys. Lett. 32, 647 (1978).

${ }^{2}$ Y. Kondo, K. Nouchi, T. Mitsuyu, M. Watanabe, P. G. Kazansky, and K. Hirao, Opt. Lett. 24, 646 (1999).

${ }^{3}$ C. Montero, C. Gomez-Reino, and J. L. Brebner, Opt. Lett. 24, 1487 (1999).

${ }^{4}$ J. Zhang, K. Sugioka, and K. Midorikawa, Opt. Lett. 23, 1486 (1998).

${ }^{5}$ G. P. Lua et al., Appl. Phys. Lett. 69, 1352 (1996).

${ }^{6}$ B. Wu, P. L. Chu, and Z. Xiong, IEEE J. Quantum Electron. 35, 1369 (1999).

${ }^{7}$ C. Y. Chao, C. Y. Chen, C. W. Liu, Y. Chang, and C. C. Yang, Appl. Phys. Lett. 71, 2442 (1997).

${ }^{8}$ J. J. Shin, S. Gurtler, Y. Chang, and C. C. Yang, Appl. Phys. Lett. 72, 2808 (1998).

${ }^{9}$ D. Hofstetter, R. L. Thornton, L. T. Romano, D. P. Bour, M. Kneissl, and R. M. Donalson, Appl. Phys. Lett. 73, 2158 (1998).

${ }^{10}$ P. S. Ramanujam, M. Pedersen, and S. Hvilsted, Appl. Phys. Lett. 74, 3227 (1999).

${ }^{11}$ Properties of Crystalline Silicon, edited by R. Hull (INSPEC, London, 1999).

${ }^{12} \mathrm{P}$. Baeri and S. U. Campisano, in Laser Annealing of Semiconductors, edited by J. M. Mayer and J. M. Poate (Academic, New York, 1982).

${ }^{13}$ P. Baeri and E. Rimini, Mater. Chem. Phys. 46, 169 (1996).

${ }^{14}$ S. Gwo, C. L. Yeh, P. F. Chen, Y. C. Chou, T. T. Chen, T. S. Chao, S. E. Hu, and T. Y. Huang, Appl. Phys. Lett. 74, 1090 (1999).

${ }^{15}$ R. B. Capaz, L. V. C. Assali, L. C. Kimerling, K. Cho, and J. D. Joannopoulos, Phys. Rev. B 59, 4898 (1999).

${ }^{16}$ Y. S. Liu, S. W. Chiang, and F. Bacon, Appl. Phys. Lett. 38, 1005 (1981).

${ }^{17}$ M. Nakamura, Y. Mochizuki, K. Usami, Y. Itoh, and T. Nozaki, Solid State Commun. 50, 1079 (1984).

${ }^{18}$ D. H. Auston, J. A. Golovchenkov, A. L. Simons, C. M. Surko, and T. N. C. Venkatesan, Appl. Phys. Lett. 34, 777 (1979).

${ }^{19}$ A. Blatter and C. Ortiz, J. Appl. Phys. 73, 8552 (1993).

${ }^{20}$ C. Ortiz and A. Blatter, Thin Solid Films 218, 209 (1992).

${ }^{21}$ M. Himmelbauer, E. Arenholz, D. Bauerle, and K. Schilcher, Appl. Phys. A: Mater. Sci. Process. 63, 337 (1996).

${ }^{22}$ K. Piglmayer, E. Arenholz, C. Ortwein, N. Arnold, and D. Bauerle, Appl. Phys. Lett. 73, 847 (1998).

${ }^{23}$ S. Chen, C. P. Grigoropoulos, H. K. Park, P. Kerstens, and A. C. Tam, Appl. Phys. Lett. 73, 2093 (1998). 